ORIGINAL PAPER

\title{
Clinicopathologic CHaRACTERistics AND Resection RATES OF PAPILLARY EARLY GASTRIC CANCER REMOVED BY ENDOSCOPIC SUBMUCOSAL DISSECTION
}

\author{
Katarzyna Karpińska-KaczmarczyK ${ }^{1}$, Magdalena Lewandowska ${ }^{1}$, Andrzej BialeK ${ }^{2}$, \\ Maegorzata ŁawniczaK $^{2}$, Ewa DobaK ${ }^{1}$, Elżbieta Urasińska ${ }^{1}$
}

${ }^{1}$ Department of Pathology, Pomeranian Medical University, Szczecin, Poland
${ }^{2}$ Department of Gastroenterology, Pomeranian Medical University, Szczecin, Poland

\begin{abstract}
The aim of this study was to assess the: 1) clinicopathologic features of papillary early gastric cancer (PEGC) (13 cases) compared to tubular early gastric cancer (TEGC) (41 cases); 2) efficiency of endoscopic submucosal dissection (ESD) in treatment of PEGC. From January 2007 to February 2016, a total of 54 consecutive patients with early gastric cancer (EGC) underwent ESD at the Department of Gastroenterology of the Pomeranian Medical University in Poland. The histologic type of carcinoma was assessed according to the WHO histological classification of GC. The extension of GC into the submucosa was measured using the Aperio Scan Scope image analysis system tools. PEGCs were diagnosed in $24.1 \%$ of the cases of EGC. PEGCs were significantly more elevated in macroscopic examination and better demarcated tumors than TEGC. There were no significant differences between gender, tumor location, ulceration, tumor size, depth of invasion (T), presence of intestinal metaplasia and lymphocytic infiltrate. Curative resection was achieved in $87.1 \%$ of patients with EGCs treated with ESD. The lower rate of curative resection (R0) observed in PEGC (76.9\%) vs TEGC (90.2\%) was not statistically significant. Further studies will be necessary to confirm the clinical and morphological presentation of PEGCs.
\end{abstract}

Key words: early gastric cancer, endoscopic submucosal dissection, papillary adenocarcinoma, resection rate.

\section{Introduction}

The World Health Organization classification of gastric carcinomas (GCs) recognizes four main histological pattern types based on the predominant histological pattern of the carcinoma: tubular, papillary, mucinous and poorly cohesive (including signet ring cell type) [1]. Most gastric cancers show significant variability, and the predominant histologic pattern is used to represent the final descriptive diagnosis [2]. Among these entities, papillary adenocarcinoma is a rare histologic variant of GC that is characterized histologically by epithelial projections scaffolded by a central fibrovascular core [1]. The invasive front of this type of GC usually is distinctly demarcated from surrounding structures [2], and a dense inflammatory component can be seen [2]. This variant accounts for between $6 \%$ and $11 \%$ of GCs and tends to affect older patients [2]. It has been commonly reported in the proximal stomach and is frequently associated with liver metastases and a higher rate of lymph node metastases [2]. The papillary variant of GC represents $1-37 \%$ of cases of early gastric cancers (EGCs) and, like the tubular variant, can be difficult to dif- 
ferentiate from dysplasia $\{2,3]$. Tubular adenocarcinoma is the most common histologic type of EGCs, representing $52 \%$ of cases [2]. This type of cancer is composed of distended and anastomosing branching tubules of various size [2]. Mucous and cellular and inflammatory debris can be noted intraluminally. Individual neoplastic cells can be either columnar or cuboidal, or with various degrees of atypia and mitosis.

Recent developments in endoscopic submucosal dissection (ESD) have enabled en bloc resection of EGC with a negligible risk of lymph node metastases irrespective of tumor size or the presence of submucosal fibrosis $[3,4,5,6]$. ESD is minimally invasive and highly curative treatment of EGC. It allows one to conserve the whole stomach with postoperative good quality of life [6]. However, relative to low incidence of EGC in Western countries, their experience with minimally invasive surgical and endoscopic methods is low compared to that of Asian countries [6,7]. It is known that some clinicopathologic features of GC are different in the two worlds and have not been thoroughly studied. There exists a limited body of literature on papillary early gastric cancer (PEGC). Biologic behavior and prognostic significance of this histological type of GC are unclear. In most papers, analysis of some clinicopathologic features or efficiency of ESD in treatment of EGC is assessed in subgroups histologically classified, which might be poorly differentiated or categorized using the Lauren classification without special focus on the papillary subtype of GC $[8,9]$. Therefore, the aim of this study was to assess the following: 1) clinicopathologic features and p53 protein immunohistochemical expression of papillary early gastric cancer (PEGC) compared to tubular early gastric cancer (TEGC); 2) efficiency of ESD in treatment of PEGC.

\section{Material and methods}

From January 2007 to February 2016, a total of 54 consecutive patients with EGCs underwent ESD at the Department of Gastroenterology of the Pomeranian Medical University in Poland. All patients underwent endoscopic ultrasound (EUS) and abdominal computed tomography before hospital admission

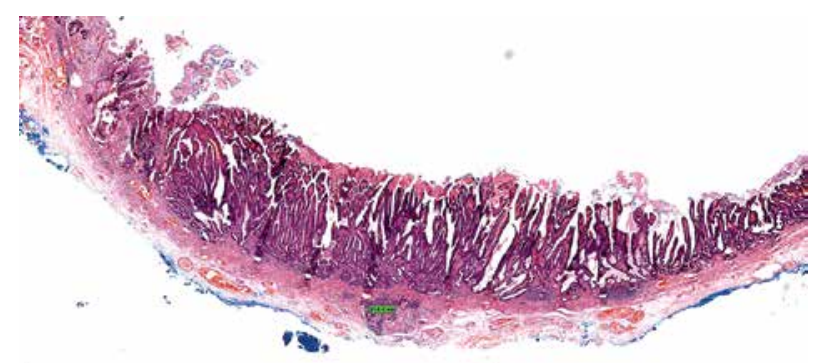

Fig. 1. Papillary early gastric cancer, HE staining: low power view (original magnification $40 \times$ ) with ESD referral. Stomach lesions were included in the study based on the expanded criteria of the Japanese Gastric Cancer Association and European Society of Gastrointestinal Endoscopy [10, 11]. After resection of the lesion, specimens were pinned on a polystyrene board to prevent rolling of the edges and to identify horizontal margins, and then fixed in $10 \%$ buffered formalin. The pathological tumor size of ESD specimens was measured and the macroscopic type of lesion was assessed according to the Paris endoscopic classification of superficial neoplastic lesions [12]. ESD specimens were serially sectioned at $2-\mathrm{mm}$ intervals and embedded in paraffin using the standard procedure. For each specimen, $3-\mu \mathrm{m}$ thick sections were prepared and used for hematoxylin and eosin (HE). Out of these 54 cases, 13 were diagnosed as PEGC (Figs. 1 and 2) and 41 as TEGC, based on the dominant histological pattern according to the WHO histological classification of GC [1]. Every specimen was evaluated by two pathologists to obtain a consensus in the final diagnosis. The presence of ulceration and lymphovascular invasion was also assessed. Cancer involvement and the level of safe lateral and vertical margins were reported. The depth of invasion ( $\mathrm{T}$ staging) was evaluated according to WHO [13]. The extension of GC into the submucosa was measured using the Aperio Scan Scope image analysis system tools (in microns) according to the guidelines of the Japanese Gastric Cancer Association [14]. In patients treated by ESD resection was regarded to be curative (R0) when tumor-free lateral and vertical margins were achieved, with the depth of submucosal invasion lower than $500 \mu \mathrm{m}$ and absence of lymphovascular invasion. Resection was regarded to be non-curative (R1) if the expanded criteria were not met and/or cancer cells were found in surgical margins. The tumor borders were described as sharp or poorly demarcated. The presence of intestinal metaplasia and lymphocytic infiltrate in the gastric mucosa in the vicinity of the tumor was also examined. A semiquantitative approach was used to score the intensity of lymphocytic infiltrate in the vicini-

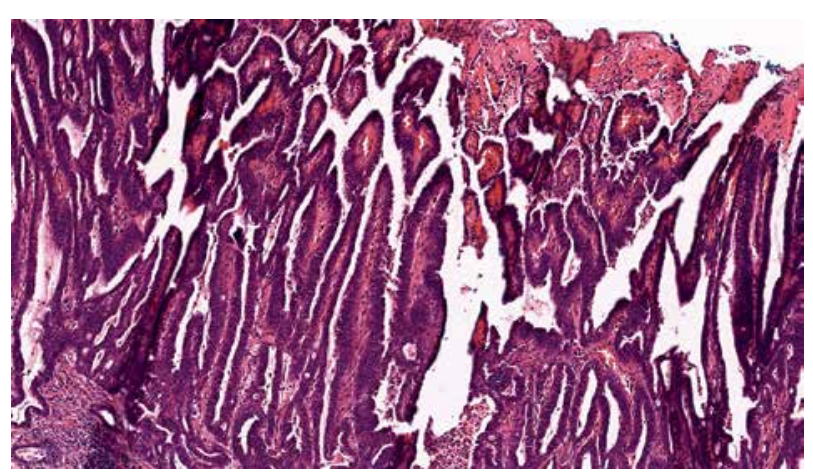

Fig. 2. Papillary early gastric cancer, HE staining: high power view (original magnification $200 \times$ ) 
ty of the tumor $(0-$ lack of lymphocytic infiltrate, 1 - mild lymphocytic infiltrate, 2 - moderate lymphocytic infiltrate, 3 - severe lymphocytic infiltrate).

\section{Statistical analysis}

Fisher's exact test for categorical variables and Student's t-test for numerical variables were used in the statistical analysis. Statistica (version 10, www. statsoft.com) software was used. A p value of $<0.05$ was considered statistically significant.

\section{Results}

Clinicopathologic features of 54 patients with EGCs treated by ESD are found in Table I.

The mean patient age was $69.6 \pm 11.0$ years (range 44-89 years). The male/female ratio was $1.25 / 1$. PEGC was diagnosed in $24.1 \%$ of examined EGCs. Macroscopically the predominant macroscopic tumor types were flat (IIb) and superficial shallow (IIc). The mean tumor size was $11.4 \pm 6.0 \mathrm{~mm}$ (range 2-30 $\mathrm{mm}$ ). In addition, muscularis mucosa invasion was confirmed in 26 cases of EGCs. In all cases classified as R1 (7 cases), submucosal invasion was noted. In $6 \mathrm{R} 1$ tumors the extension of GC into the submucosa exceeded $500 \mu \mathrm{m}$, and in 3 cases malignant tissue was found in the vertical margin. Curative resection was obtained in $87.1 \%$ of patients. None of the patients had metastases in regional lymph nodes. No vascular invasion was found. Table II reports correlations between clinicopathologic features, resection rates and histological types of EGC (PEGC vs. TEGC).

Univariate analysis revealed that PEGC compared to TEGC was significantly more commonly macroscopically presented as elevated lesions with sharp (well-demarcated) tumor borders.

Although not statistically significant, the depth of submucosal invasion in PEGC was 2.5 times higher than that of TEGC $(1323.8 \mu \mathrm{m}$ in PEGC vs. 521.54 $\mu \mathrm{m}$ in TEGC). A depth of submucosal invasion that exceeded $500 \mu \mathrm{m}$ was found in $25 \%$ cases of PEGC (3/13) vs. $7.3 \%$ cases of TEGC (3/41). In 1 case of PEGC and in 2 cases of TEGCs neoplastic infiltration was found in vertical margins. Despite the higher percentage of muscularis mucosa invasion in $\mathrm{pT} 1 \mathrm{a}$ PEGC and deeper submucosal invasion, R0 was not significantly lower in the papillary subtype of EGC compared to the tubular subtype (76.9\% vs. $90.2 \%)$.

\section{Discussion}

ESD is an effective and safe method for resection of neoplastic gastric lesions, with a low recurrence rate $[5,6,15,16,17]$. Despite good treatment results, ESD is still rarely used in Europe, but is progressively gaining more attention in other Western countries $[11,16,17]$. The improvement of endo-
Table I. Clinicopathologic features of 54 patients with EGCs

\begin{tabular}{ll}
\hline Parameter & No. $(\%)$ \\
\hline Age (years) & $69.6 \pm 11.0$ \\
\hline Gender & $30(55.5 \%)$ \\
\hline male & $24(44.5 \%)$ \\
\hline female & $13(24.1 \%)$ \\
\hline Histological type & $41(75.9 \%)$ \\
\hline papillary & \\
\hline Lubular & $13(24.1 \%)$ \\
\hline upper & $16(29.6 \%)$ \\
\hline middle & $25(46.3 \%)$ \\
\hline lower &
\end{tabular}

Macroscopic type

\begin{tabular}{lc}
\hline 0-Ip & $6(11.1 \%)$ \\
\hline $0-$ Is & $12(22.2 \%)$ \\
\hline $0-$ IIa & $6(11.1 \%)$ \\
\hline $0-$ IIb & $23(42.6 \%)$ \\
\hline 0-IIc & $8(14.8 \%)$ \\
\hline Ulcer & $5(9.3 \%)$ \\
\hline present & $49(90.7 \%)$ \\
\hline absent & \\
\hline
\end{tabular}

Tumor size

\begin{tabular}{ll}
\hline$\leq 10 \mathrm{~mm}$ & $24(44.4 \%)$ \\
\hline$>10 \mathrm{~mm}$ & $30(55.6 \%)$ \\
\hline
\end{tabular}

Depth of invasion $-\mathrm{T}$

\begin{tabular}{ll}
\hline T1a & $42(77.8 \%)$ \\
\hline T1b & $12(22.2 \%)$
\end{tabular}

Muscularis mucosa invasion

\begin{tabular}{ll}
\hline negative & $28(51,9 \%)$ \\
\hline positive & $26(48.1 \%)$ \\
\hline
\end{tabular}

Intestinal metaplasia

\begin{tabular}{ll}
\hline negative & $20(37.1 \%)$ \\
\hline positive & $34(62.9 \%)$
\end{tabular}

Lymphocytic infiltrate

\begin{tabular}{lc}
\hline 0 & $9(16.6 \%)$ \\
\hline 1 & $24(44.4 \%)$ \\
\hline 3 & $9(16.6 \%)$ \\
\hline Vascular invasion & $12(22.2 \%)$ \\
\hline negative & $54(100 \%)$ \\
\hline positive & $0(0 \%)$ \\
\hline
\end{tabular}

\begin{tabular}{lc}
\hline Resection rates & \\
\hline R0 & $47(87.1 \%)$ \\
\hline R1 & $7(12.9 \%)$ \\
\hline
\end{tabular}

$R 0$ - curative resection; $R 1$ - non-curative resection 
Table II. Clinicopathologic features, resection rates in PEGC vs. TEGC

\begin{tabular}{|c|c|c|c|}
\hline Parameter & $\begin{array}{c}\text { PEGC } \\
(\mathrm{N}=13) \\
\text { No. }(\%)\end{array}$ & $\begin{array}{c}\text { TEGC } \\
(\mathrm{N}=41) \\
\text { No. }(\%)\end{array}$ & $\mathrm{P}$ \\
\hline Age (mean) years & $72.5 \pm 9.9$ & $68.7 \pm 11.3$ & NS \\
\hline \multicolumn{4}{|l|}{ Gender } \\
\hline male & $8(61.5 \%)$ & $22(53.7 \%)$ & \multirow[t]{2}{*}{ NS } \\
\hline female & $5(38.5 \%)$ & $19(46.3 \%)$ & \\
\hline \multicolumn{4}{|l|}{ Location of tumor } \\
\hline upper & $6(46.2 \%)$ & $7(17.1 \%)$ & \multirow[t]{2}{*}{ NS } \\
\hline middle, lower & $7(53.8 \%)$ & $34(82.9 \%)$ & \\
\hline \multicolumn{4}{|l|}{ Macroscopic type } \\
\hline 0-Ip, 0-Is, O-IIa & $10(76.9 \%)$ & $14(34.1 \%)$ & \multirow[t]{2}{*}{0.01} \\
\hline 0-IIb, 0-IIc & $3(23.1 \%)$ & $27(65.9 \%)$ & \\
\hline \multicolumn{4}{|l|}{ Ulcer } \\
\hline present & $1(7.7 \%)$ & $4(9.7 \%)$ & \multirow[t]{2}{*}{ NS } \\
\hline absent & $12(92.3 \%)$ & $37(90.3 \%)$ & \\
\hline \multicolumn{4}{|l|}{ Tumor size } \\
\hline$\leq 10 \mathrm{~mm}$ & $5(38.5 \%)$ & $19(46.3 \%)$ & \multirow[t]{2}{*}{ NS } \\
\hline$>10 \mathrm{~mm}$ & $8(61.5 \%)$ & $22(53.7 \%)$ & \\
\hline \multicolumn{4}{|l|}{ Tumor borders } \\
\hline sharp & $11(84.6 \%)$ & $17(41.5 \%)$ & \multirow[t]{2}{*}{0.01} \\
\hline poorly defined & $2(15.4 \%)$ & $24(58.5 \%)$ & \\
\hline \multicolumn{4}{|c|}{ Depth of invasion $-\mathrm{T}$} \\
\hline T1a & $8(61.5 \%)$ & $34(82.9 \%)$ & \multirow[t]{2}{*}{ NS } \\
\hline $\mathrm{T} 1 \mathrm{~b}$ & $5(38.5 \%)$ & $7(17.1 \%)$ & \\
\hline \multicolumn{4}{|c|}{ Muscularis mucosa invasion } \\
\hline absent & $4(30.8 \%)$ & $24(58.5 \%)$ & \multirow[t]{2}{*}{ NS } \\
\hline present & $9(69.2 \%)$ & $17(41.5 \%)$ & \\
\hline \multicolumn{4}{|l|}{ Intestinal metaplasia } \\
\hline negative & $3(23.1 \%)$ & $17(41.5 \%)$ & \multirow[t]{2}{*}{ NS } \\
\hline positive & $10(76.9 \% 0$ & $24(58.5 \%)$ & \\
\hline \multicolumn{4}{|c|}{ Lymphocytic infiltrate } \\
\hline $0+1$ & $8(61.5 \%)$ & $25(60.9 \%)$ & \multirow[t]{2}{*}{ NS } \\
\hline $2+3$ & $5(38.5 \%)$ & $16(39.01 \%)$ & \\
\hline \multicolumn{4}{|l|}{ Resection } \\
\hline R0 & $10(76.9 \%)$ & $37(90.2 \%)$ & \multirow[t]{2}{*}{ NS } \\
\hline R1 & $3(32.1 \%)$ & $4(9.8 \%)$ & \\
\hline
\end{tabular}

PEGC - papillary early gastric cancer; TEGC - tubular early gastric cancer; $R O$ - curative resection; $R 1$ - non-curative resection

scopic techniques and better knowledge of the problem have led to significant increase in EGC over the past few years [18]. According to different screening programs in Japan and Korea about fifty percent of treated patients had an early tumor stage $[6,18]$. The prevalence of EGC in Western countries is not really known. There are no screening programs for this tumor entity [6]. Some European data registered a $14.5-37.8 \%$ rate of T1 stage of GC [6].

Few reports have analyzed clinicopathologic characteristics of papillary adenocarcinoma because it is a rare histologic entity among GCs $[3,8,15,19,20$,
21]. In the literature PEGC represents from $1 \%$ to $37 \%$ of cases of EGCs $[2,3,6]$. In the present study PEGCs were diagnosed in $24.1 \%$ of all EGCs. The clinicopathologic characteristics of the diagnosed EGCs revealed that PEGCs grossly were found as elevated, sharply demarcated lesions. Half of PEGCs were located in the upper stomach, but there was no significant difference in location or histological type of EGC. Lee et al. [3], in contrast to most trials, reported that papillary adenocarcinoma-type EGCs were mostly located in the lower third of the stomach but agreed with elevated macroscopic shape. The location of lesions in the upper part of the stomach, their size, and the presence of submucosal fibrosis are associated with longer procedure duration, a lower resection rate and a higher rate of complications [17]. In this study, in 6 out of 13 EGCs (3 cases of PEGC/ 3 cases of TEGC) that were located in the upper stomach, submucosal invasion was found. In the present study, R0 was achieved in $87.1 \%$ of cases, but comparison between PEGC and TEGC (76.9\% vs. $90.2 \%$ ) suggests that the papillary histological type may potentially reduce the efficiency of ESD in treatment of EGCs. Interestingly, the univariate analysis revealed no significant association. In our study PEGCs compared to TEGCs showed more common and deeper submucosal invasion $(38.5 \%$ of cases of PEGCs vs. $17.1 \%$ of cases of TEGCs). Submucosal invasion exceeding $500 \mu \mathrm{m}$ was found to be the most important factor for non-curative resection (R1). This study demonstrated that T1a papillary GCs more commonly than TEGC (although not statistically significantly) infiltrated muscularis mucosa, which additionally confirmed the more aggressive nature of those tumors. In data published by Lee et al., more than half of patients who underwent ESD as primary treatment for PEGC ultimately achieved out-of-ESD indication [3]. Kodama et al. reported that PEGC characterized the PEN A subtype (expanding carcinoma) of submucosal invasion with high incidence of lymphogenous metastases, recurrences and poorer prognosis compared to the super type or PEN B type which are found in tubular and poorly differentiated carcinomas [10]. Some studies have reported that a papillary adenocarcinoma component in EGC was associated with a higher risk of lymphatic involvement and a higher percentage of lymph node metastases $[3,20]$. The present study clearly demonstrated that ESD used for treatment of PEGC should be implemented more carefully, because of the more invasive character of this histological type of GC.

This study is limited by its retrospective design and the small sample size associated with the relative rarity of PEGC. Determining the most appropriate prevalence of the papillary subtype of EGC in the pathological diagnosis of $\mathrm{GC}$ will be important in future [3]. However, further studies will be necessary 
to confirm the clinical and morphological presentation of diagnosed cases of the papillary type of EGCs.

\section{Conclusions}

Based on the presented data, complete removal of EGC was achieved in about $87 \%$ of all cases, confirming that ESD is an effective therapeutic approach. Our results are comparable with those of larger Asian series, suggesting that ESD used for treatment of EGC should be implemented in more European centers. This study showed that PEGCs were significantly more elevated in macroscopic examination and better demarcated tumors than TEGCs. There were no statistically significant differences between gender, tumor location, ulceration, tumor size, depth of invasion (T), intestinal metaplasia, lymphocytic infiltrate, $\mathrm{p} 53$ expression and resection rate.

\section{The authors declare no conflict of interest.}

\section{References}

1. Bosman FT, Carneiro F, Hruban RH, et al. WHO Classification of Tumors of the Digestive System. IARC, Lyon 2010.

2. Tan D, Lauwers GY. Advances in surgical pathology: Gastric cancer. Lippincott Williams and Wilkins, Philadelphia, 2010.

3. Lee HJ, Kim GH, Park do Y, el al. Is endoscopic submucosal dissection safe for papillary adenocarcinoma of the stomach? World J Gastroenterol 2015; 21: 3944-3952.

4. Herreros de Tejada A. ESD training: A challenging path to excellence. World J Gastrointest Endosc 2014; 6: 112-120.

5. Espinel J, Pinedo E, Ojeda V, et al. Treatment modalities for early gastric cancer. World J Gastrointest Endosc 2015; 7: 1062-1069.

6. Bollschweiler E, Berlth F, Baltin Ch, et al. Treatment of early gastric cancer in the Western World. World J Gastroenterol 2014; 20: 5672-5678.

7. Othman MO, Wallace MB. Endoscopic mucosal resection (EMR) and endoscopic submucosal dissection (ESD) in 2011, a Western perspective. Clin Res Hepatol Gastroenterol 2011; 35: 288-294.

8. Takizawa K, Ono H, Kakushima N, et al. Risk of lymph node metastases from intramucosal gastric cancer in relation to histological types: how to manage the mixed histological type for endoscopic submucosal dissection. Gastric Cancer 2013; 16: 531-536.

9. Lauren P. The two histological main types of gastric carcinoma: diffuse and so-called intestinal - type carcinoma. An attempt at a histo-clinical classification. Acta Pathol Microbiol Scand 1965; 64: 31-49.

10. Kodama Y, Inokuchi K, Soejima K, et al. Growth patterns and prognosis in early gastric carcinoma. Superficially spreading and penetrating growth types. Cancer 1983; 51: 320-326.

11. Japanese Gastric Cancer Association. Japanese gastric cancer treatment guidelines 2010. Gastric Cancer 2011; 14: 113-123.

12. Endoscopic Classification Review Group. Update on the Paris classification of superficial neoplastic lesions in the digestive tract. Endoscopy 2005; 37: 570-578.

13. Rhodes TD, Harris JE. TNM Classification for Gastric Cancer. Updated Jan 17, 2014. 2011; 35: 288-294.
14. Japanese Gastric Cancer Association. Japanese classification of gastric carcinoma: 3rd English edition. Gastric Cancer 2011; 14: 101-112.

15. Pimentel-Nunes P, Dinis-Ribeiro M, Ponchon T, et al. Endoscopic submucosal dissection: European Society of Gastrointestinal Endoscopy (ESGE) Guideline. Endoscopy 2015; 47 : 829-854.

16. Repici A, Zullo A, Hassan C, et al. Endoscopic submucosal dissection of early gastric neoplastic lesions: a western series. Eur J Gastroenterol Hepatol 2013; 25: 1261-1264.

17. Białek A. Wiechowska-Kozłowska A, Pertkiewicz J, et al. Endoscopic submucosal dissection for the treatment of neoplastic lesions in the gastrointestinal tract. World J Gastroenterol 2013; 19: 1953-1961.

18. Saragoni L, Morgagni P, Gardini A, et al. Early gastric cancer: diagnosis, staging, and clinical impact. Evaluation of $530 \mathrm{pa}-$ tients. New elements for an updated definition and classification. Gastric Cancer 2013; 16: 549-554.

19. Yasuda K, Adachi Y, Shiraishi N, et al. Papillary adenocarcinoma of the stomach. Gastric Cancer 2000; 3: 33-38.

20. Sekiguchi M, Kushima R, Oda I, et al. Clinical significance of a papillary adenocarcinoma component in early gastric cancer: a single-center retrospective analysis of 628 surgically resected early gastric cancers. J Gastroenterol 2015; 50: 424-434.

21. Kanemitsu T, Yao K, Nagahama T, et al. The vessels within epithelial circle (VEC) pattern as visualized by magnifying endoscopy with narrow-band imaging (ME-NBI) is a useful marker for the diagnosis of papillary adenocarcinoma: a case-controlled study. Gastric Cancer 2014; 17: 469-477.

\section{Address for correspondence}

Katarzyna Karpińska-Kaczmarczyk

Department of Pathology

Pomeranian Medical University

Unii Lubelskiej 1

71-252 Szczecin, Poland

tel. +48914253502

fax: +48914870032

e-mail:kkarp@pum.edu.pl 